\title{
Mechanism of transformation of protons in the process of creating aqueous-alcoholic mixtures
}

\section{Oleg Kuzmin}

\author{
National University of food Technologies, Kyiv, Ukraine
}

Keywords:

Ethanol

Water

Mixture

${ }^{1} \mathrm{H}$ NMR

Stabilization

\section{Article history:}

Received

13.10.2017

Received in revised

form 25.12.2017

Accepted

29.12.2017

\section{Corresponding author:}

Oleg Kuzmin

E-mail:

kuzmin_ovl@

nuft.edu.ua

\section{DOI:}

$10.24263 / 2304-$

974X-2017-6-4-9

\section{Abstract}

Introduction. The aim of the publication is to study the mechanisms of transformation of ethanol protons (ethyl rectified spirit - ERS) and water (drinking water) in the process of creating aqueous-alcoholic mixtures (AAM) by ${ }^{1} H N M R$ spectroscopy.

Materials and methods. ${ }^{1} H N M R$ analysis was conducted with the usage of the following: $F T-N M R$ Bruker Avance II spectrometer with operating frequency at ${ }^{1} \mathrm{H}-400 \mathrm{MHz}$; specially shaped capillary with acetone- $d_{6}$ (atomic fraction of deuterium $99,88 \%$; chemical shift of the residual proton ${ }^{l} H-\delta=2,75 \mathrm{ppm}$ ); high accuracy ampoules № 507-HP for high resolution $N M R$ 's spectroscopy (400 MHz); volumetric pipette; dispenser; ERS; drinking water; AAM from ERS and drinking water.

Work methodology: $0,3 \mathrm{ml}$ of a AAM prepared with a volumetric pipette with a predetermined strength $(40,0 \pm 0,2) \%$ vol.; external standard separated from the testing substance which is required for LOCK's system operation (acetone- $d_{6}$ ) of $N M R^{\text {'s }} \mathrm{s}$ deuterium stabilization spectrometer) is added in a special form of a capillary into an ampoule. The obvious advantage of using the external standard is the fact that standard substance's molecules and test's solution do not interact with each other, ${ }^{l} H$ NMR spectra records and data processing were performed according to the instruction of FT-NMR Bruker Avance II spectrometer (400 $\mathrm{MHz}$ ).

Results and discussion. In this paper, we have established fundamentally new features in the process of creating AAM that are directly dependent on the time of contact with water and ERS. As a results we have evidence of a complex dynamic of achievement processes of solution equilibrium for AAM prepared in drinking water with $\mathrm{pH}=7,01$ and ERS. At the same time $\mathrm{pH}$ of obtained AAM is $\mathrm{pH}=8,32$. Hydroxyl proton of ethanol (EtOH) exchange rate is in intermediate area. It happens during the first 48 hours when the concentration of alcohol is constant (strength AAM - 39,94\% vol.) and the system is thermostatic $\left(\mathrm{t}=23,5^{\circ} \mathrm{C}\right)$. Signals are separately located. Protons' exchange is accelerated due to the rearrangement of system's structure, during the interval of $\tau=48 \mathrm{~h}$ to $120 \mathrm{~h}$. Since $\tau=120 \mathrm{~h}$, there is only one common signal of mobile protons of asymmetrical shape. The size of chemical shift of the summed signal $\delta_{E t O H+H 2 O}=4,74 \mathrm{ppm}(\tau=120 \mathrm{~h})$ is starting to grow and shifts to the «weak fields» with the value of $\delta_{E t O H+H 2 O}=4,81 \mathrm{ppm}(\tau=312 \mathrm{~h})$.

Conclusion. In this paper, we have established fundamentally new features in the process of creating AAM that are directly dependent on the time of contact with drinking water and ERS. 


\section{Introduction}

$N M R$ spectroscopy is widely used in physics research, industry, agriculture and other industries. $N M R$ plays a particularly important role in food chemistry where it used in the study of both simple organic molecules and complex macromolecular structures and their complexes (Singh, Blümich, 2016; Hore, 2017) [1, 2]. A large number of articles discuss the use of $N M R$ for research of food products; meat, fish, dairy products, vegetables, fruits, juices, pastry, cheese and alcohol products (Youssouf et al, 2017; Campo et al, 2016; Zhu, 2017; Yuan et al, 2017; Diop et al 2012; Tian et al, 2017, Sucupira et al, 2017, Li et al, 2017; Shumilina et al, 2016; Okaru et al, 2017) [3-12]. This method provides comprehensive information with relatively simple obtaining spectra, thus greatly facilitating and accelerating chemical research (Nose et al, 2005; Richards, Hollerton, 2011; Roberts, 2002; Hu et al, 2010) [13-16].

$N M R$ spectroscopy is most commonly applied to the nuclei of lightest isotope of hydrogen ${ }^{l} H$ (protium, ${ }^{l} H$ isotope) proton. The spectra measured using such nuclei are called proton magnetic resonance $(P M R)$ spectra. $P M R$ accounts for about $90 \%$ of all research on NMR spectra. Most of them operate in the Fourier transform mode (Richards, Hollerton, 2011; Roberts, 2002) [14-15]. The principle of NMR spectroscopy is based on the magnetic properties of certain atomic nuclei that resonate in the radio frequency range of the electromagnetic spectrum when placed in a strong magnetic field at a certain magnetic field. This allows for the identification of nuclei in different chemical environments (Richards, Hollerton, 2011; Roberts, 2002) [14-15]. This property is explained by the existence of nuclei with non-zero spin (intrinsic mechanical torque), that is determined by the sum of the spins of its constituent protons and neutrons (Richards, Hollerton, 2011) [14].The spin of the isotopes' nuclei with an even number of protons and an even number of neutrons is always equal to zero (zero moment). NMR is not observed in these nuclei (Roberts, 2002) [15].

The first ${ }^{l} \mathrm{H} N M R$ spectra of ethanol $\left(\mathrm{C}_{2} \mathrm{H}_{5} \mathrm{OH}\right)$ were developed in 1951 (Arnold et al. 1951) [17]. The first ${ }^{1} \mathrm{H} N M R$ spectra of water $\left(\mathrm{H}_{2} \mathrm{O}\right)$ were obtained in 1946 (Bloch et al. 1946) [18]. At the first glance, it may seem that these are fairly simple organic molecules, at the same time $N M R$ spectroscopy exhibits grate variety (Nose et al, 2005; Richards, Hollerton, 2011; Roberts, 2002; Hu et al, 2010) [13-16] in such characteristics as chemical shift, spin-spin interactions and the effect of chemical exchange (Roberts, 2002; Matsugami et al, 2016; Jora et al, 2017) [15, 19, 20].

An ethanol molecule consists of 6 protons located in a 3 proton-containing groups: methyl $\left(\mathrm{CH}_{3}\right)$, methylene $\left(\mathrm{CH}_{2}\right)$ and hydroxyl $(\mathrm{OH})$ with a relative intensity characteristic $\mathrm{CH}_{3}: \mathrm{CH}_{2}: \mathrm{OH}-3: 2: 1$. Nuclear spin-spin interaction is observed between the three protoncontaining groups of ethanol, all of which have different resonant frequencies (Roberts, 2002) [15]. " $N$ " number of equivalent protons of one group split the signal of the nearest group into $(n+1)$ lines with the intensity of a Pascal triangle (Richards, Hollerton, 2011) [14]. The ability to observe spin-spin interactions depends on the rate of the intermolecular proton exchange (Jora et al, 2017) [20]. Wherein the hydroxyl proton $(\mathrm{OH})$ of ethanol can interchange with free hydrogen ions (Matsugami et al, 2016) [19]. The hydrogen ions are generated due to self-dissociation of water or traces of acids, alkalis or dissociated ethanol (Jora et al, 2017) [20]. The concentration of free ions is characterized by $\mathrm{pH}$ level.

Vodka - is an alcoholic drink with strength from $37,5 \%$ to $56 \%$, obtained by mixing ERS with water and treated with activated carbon, with addition of non-volatile ingredients or without them.

In the opinion Hu et al. (2010) [16] vodka is a fairly simple physicochemical system: a 
mixture of alcohol and water. However, each brand has its own distinctive taste and features on the molecular level. Research conducted by Hu et al. (2010) [16] confirm that these differences are significant both during the stage of creating AAM, and in the final product - the commercial vodka. The major differences are associated with hydrogen bonds, in particular their strength, as confirmed by various research methods such as ${ }^{1} \mathrm{H}$ NMR spectroscopy, FTIR spectroscopy, Raman spectroscopy. ${ }^{l} H$ NMR and FTIR spectroscopy demonstrates the presence of water in the hydrate structure $\mathrm{EtOH} \cdot(5,3 \pm 0,1) \mathrm{H}_{2} \mathrm{O}$. Water can also be observed in AAM as well as in vodka. The authors (Hu et al, 2010) [16] attribute this value with the perception of organoleptic characteristics of vodka.

In their paper, the authors $(\mathrm{Hu}$ et $\mathrm{al}, 2010)$ [16] introduced the concept of «structurability» - defined as the ability to maintain structure - a parameter that determines the ability of vodka (alcohol) to streamline its structure.

The effect of impurities (such as salts, acids, phenols) strengthening the hydrogen bonds in AAM as well as in the finished product such as sake, has been studied by Nose et al (2005) [13]. Hu et al [16] have identified that the impurity of compounds has an effect on the molecular dynamics in ethanol's hydration process.

Previously, we have conducted primary research of ${ }^{l} H$ NMR AAM, which were described in the work of Kuzmin et al, 2013-2017 [21-24]. The obtained results give grounds to assert a fundamental difference in the behavior of the AAM prepared from the alcohol and water passing through various processes. This may indicate the presence of such features as separate signals of hydroxyl protons of $\mathrm{H}_{2} \mathrm{O}$ and $\mathrm{EtOH}$. Also abnormal waveforms of $\mathrm{CH}_{3}$ and $\mathrm{CH}_{2}$ characterize a product with a lower tasting properties. The presence of the combined signal of $\mathrm{EtOH}+\mathrm{H}_{2} \mathrm{O}$ and rational form of $\mathrm{CH}_{3}$ and $\mathrm{CH}_{2}$ signals (triplet - for $\mathrm{CH}_{3}$, quartet - for $\mathrm{CH}_{2}$ ) - characterizes the AAM with the best tasting properties.

Thus, in the work of Kuzmin O., Sujkov S. et al, 2013 [21] established experimental evidence of instalment nature / (non- instalment) of thermodynamic balance, taking into account the organoleptic characteristics of AAM in dependence on water treatment method and time of system's functioning. However, the questions related to internal mechanism's specification and the rate of establishment of thermodynamic balance depending on type of water used in the process of creating the AAM are remain unsolved.

Therefore, the additional research is required for a detailed study of internal mechanism of thermodynamic balance and insurance in obtaining high quality vodka products - for each type of water separately.

Therefore, the aim of this work is to study the mechanisms of transformation of ethanol protons (ERS) and water (drinking water) in the process of creating AAM by ${ }^{l} H N M R$ spectroscopy.

\section{Materials and methods}

The following characteristics of drinking water were determined: solid residual -867 $\mathrm{mg} / \mathrm{dm}^{3}$; electrical conductivity $-1150 \mu \mathrm{S} / \mathrm{cm} ; \mathrm{pH}-7,01$; redox $(\mathrm{ORP})-\ll+» 271 \mathrm{mV}$; total hardness $-7,93 \mathrm{mM} / \mathrm{dm}^{3}$; permanganate oxidation - 4,27 $\mathrm{mg} \mathrm{O} \mathrm{O}_{2} / \mathrm{dm}^{3}$; mass concentration (MC) of sodium $-90,75 \mathrm{mg} / \mathrm{dm}^{3}$; MC of potassium $-4,87 \mathrm{mg} / \mathrm{dm}^{3} ; \mathrm{MC}$ of ammonium $-<2,0 \mathrm{mg} / \mathrm{dm}^{3} ; \mathrm{MC}$ of calcium $-106,03 \mathrm{mg} / \mathrm{dm}^{3} ;$ MC of magnesium $-23,91$ $\mathrm{mg} / \mathrm{dm}^{3}$; total alkalinity $-5,38 \mathrm{mM} / \mathrm{dm}^{3}$.

Characteristics of ERS: volume part of ethanol - 96,37\%, volume part of water $3,63 \%$; content of aldehydes in anhydrous alcohol (a.a.), based on acetaldehyde $-1,3$ 
$\mathrm{mg} / \mathrm{dm}^{3}$, content of fusel oils in a.a.: propyl, isopropyl, butyl, isobutyl and isoamyl - 1,5 $\mathrm{mg} / \mathrm{dm}^{3}$; content of esters in a.a., based on of ethyl acetate $1,3 \mathrm{mg} / \mathrm{dm}^{3}$; methanol content in the a.a. $-0,0022$ vol. $\%$.

AAM sample of ERS and drinking water has the following physicochemical and organoleptic characteristics: alcoholic strength $-39,94 \%$ vol.; electrical conductivity - 183 $\mu \mathrm{S} / \mathrm{cm}$; ORP - «+» $37 \mathrm{mV}$; $\mathrm{pH}-8,32$; aldehyde content in a.a., based on acetaldehyde $1,5 \mathrm{mg} / \mathrm{dm}^{3}$; content of fusel oils in a.a.: propyl, isopropyl, butyl, isobutyl and isoamyl $-1,1$ $\mathrm{mg} / \mathrm{dm}^{3}$; the content of esters in a.a., based on acetic acid ethyl ester $-1,2 \mathrm{mg} / \mathrm{dm}^{3}$; methanol content in a.a. $-0,0022$ vol. \%; alkalinity $-2,5 \mathrm{~cm}^{3}$ of $0,1 \mathrm{M}$ hydrochloric acid for titration of $100 \mathrm{~cm}^{3}$ of AAM; oxidation test - 13,5 min; tasting score - 9,43 points (appearance - colorless liquid with residue; smell - sharp alcohol; flavor - heavy).

${ }^{1} H N M R$ analysis of AAM was conducted with the usage of the following: FT-NMR Bruker Avance II spectrometer with operating frequency at ${ }^{9} \mathrm{H}-400 \mathrm{MHz}$ (measurement error of the chemical shifts for ${ }^{I} \mathrm{H} \pm 0,0005 \mathrm{ppm}$; 5-mm broadband inverse probe with Zgradient; thermostatic system $\left(+25^{\circ} \mathrm{C} \ldots+100^{\circ} \mathrm{C}\right)$ ); specially shaped capillary with acetone$\mathrm{d}_{6}\left(\mathrm{CD}_{3}\right)_{2} \mathrm{CO}$ (atomic fraction of deuterium $-99,88 \%$; chemical shift of the residual proton $\left.{ }^{I} H-\delta=2,75 \mathrm{ppm}\right)$; high accuracy ampoules № 507-HP for high resolution NMR's spectroscopy (400 MHz); volumetric pipette; dispenser; ERS; drinking water; AAM from ERS and drinking water.

Experimental studies of ${ }^{l} H N M R$ were carried out in the following order:

- preparation of AAM;

- recording of the AAM ${ }^{1} H N M R$ spectrum;

- conclusion and interpretation of work results.

Work methodology (Kuzmin et al, 2013-2017) [21-24]:

$-0,3 \mathrm{ml}$ of a AAM prepared with a volumetric pipette with a predetermined strength $(40,0 \pm 0,2) \%$ vol.;

- external standard separated from the testing substance which is required for LOCK's system operation (acetone- $\mathrm{d}_{6}$ ) of $N M R^{\star} \mathrm{s}$ deuterium stabilization spectrometer) is added in a special form of a capillary into an ampoule. The obvious advantage of using the external standard is the fact that standard substance's molecules and test's solution do not interact with each other;

$-{ }^{I} H N M R$ spectra records and data processing were performed according to the instruction of FT-NMR Bruker Avance II spectrometer (400 MHz).

\section{Results and discussions}

Will examine spectrum of water (Figure 1), ERS (Figure 2), AAM (Figures 3-4), made of drinking water and ERS at a different instants of system's operation (life after mixing) (h).

We will examine spectra of drinking water, which is characterized by a unitary signal of hydroxyl group of $\mathrm{H}_{2} \mathrm{O}$ (Figure 1). The component of protons of $\mathrm{H}_{2} \mathrm{O}-\operatorname{singlet}(s)$, located in a «weak field» with a chemical shift $\delta_{\mathrm{H}_{2} \mathrm{O}}=4,60 \mathrm{ppm}$. Waveform of $\mathrm{H}_{2} \mathrm{O}$ protons - is distorted Gaussian curve, with a broadened base and a slight asymmetry of apex, which is offset from the centerline.

We will analyze the spectra of ERS (Figure 2). Hydroxyl group of protons of ERS are represented by two separate peaks. A component of ethanol is represented as a single broad singlet, located in a «low field» with the chemical shift $\delta_{E t O H}=5,65 \mathrm{ppm}$. A component of water proton is represented as singlet with a chemical shift of $\delta_{H 2 O}=4,85 \mathrm{ppm}$. The form of $\mathrm{H}_{2} \mathrm{O}$ protons' signal is a distorted Gaussian curve, with a broadened base and a certain asymmetry. The difference between the $\mathrm{OH}$-proton of ethanol $(\mathrm{EtOH})$ and the proton of water $\left(H_{2} \mathrm{O}\right)$ in the chemical shifts $-\Delta \delta_{I}=0,80 \mathrm{ppm}\left(\Delta f_{l}=320 \mathrm{~Hz}\right)$. 


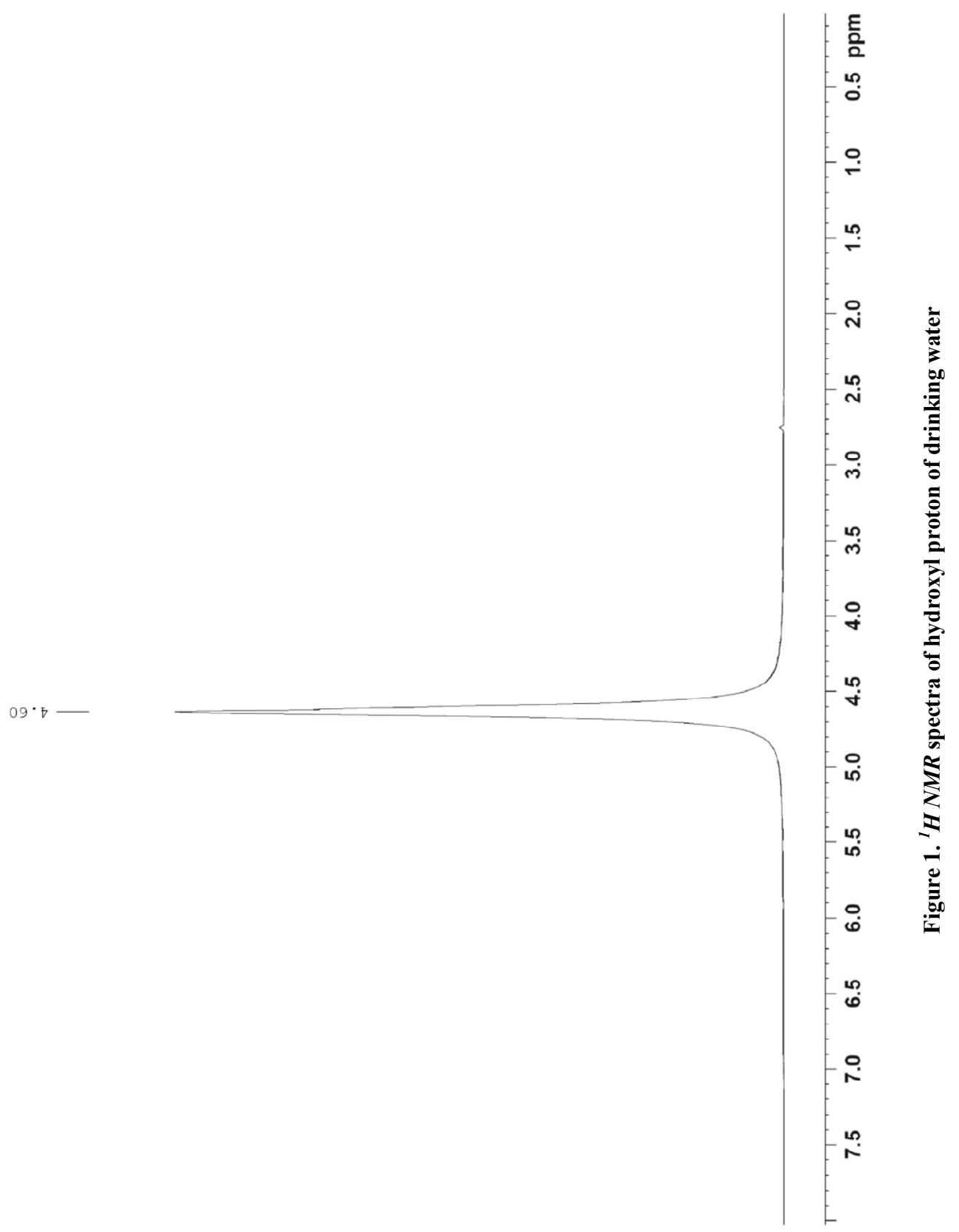




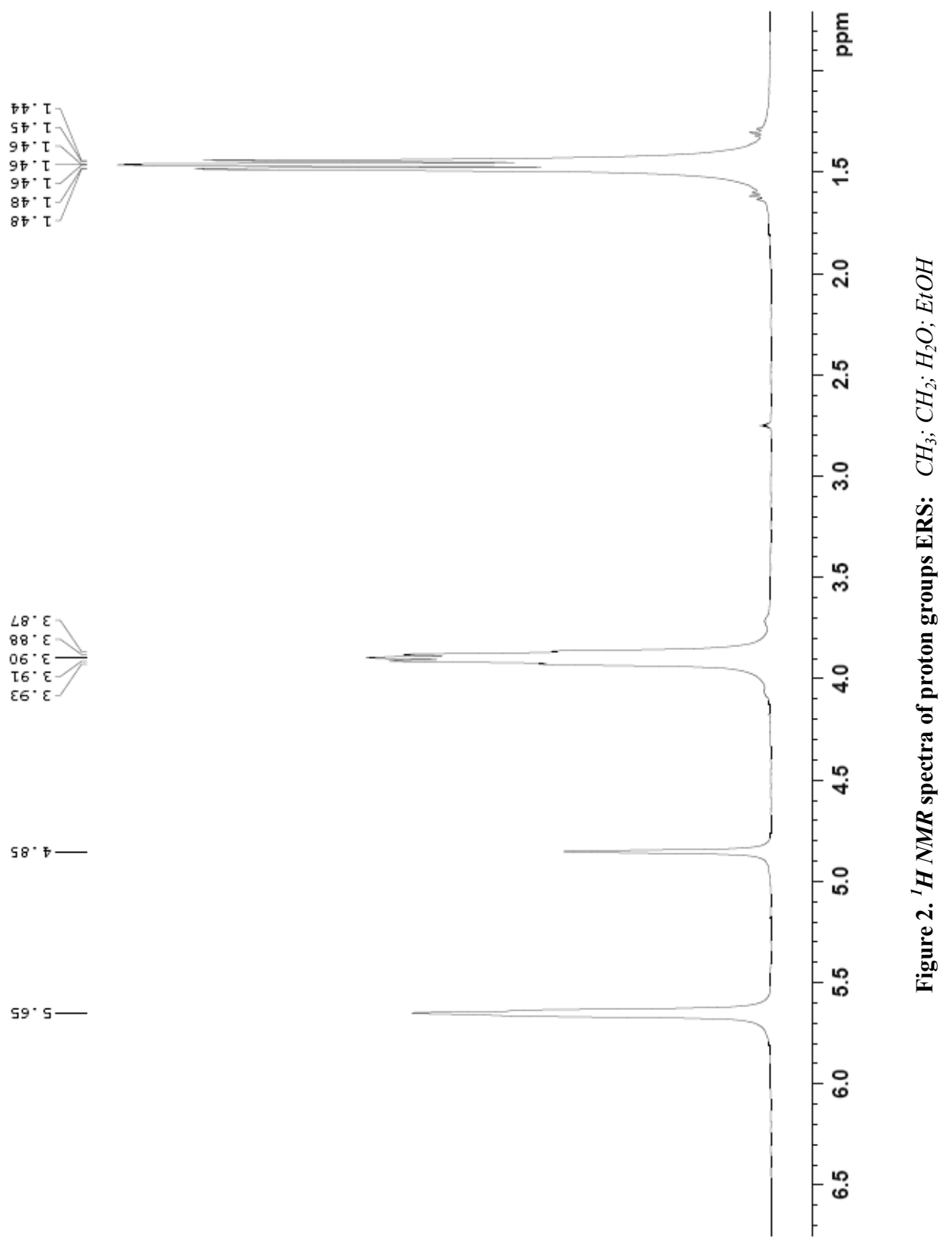


The analysis of the ${ }^{1} H N M R$-spectra of protons methyl group of ethanol $\left(\mathrm{CH}_{3}\right)$ allows us to state the following. The protons' methyl group is represented as a septet $(s p)$ with a relative intensity $(1: 6: 15: 20: 15: 6: 1)$. This is abnormity as according to Pascal's triangle and on the assumption of protons' methyl group spin-spin interactions, methylene group's $\left(\mathrm{CH}_{3}\right)$ signal has to be split by an adjacent protons' group $\left(\mathrm{CH}_{2}\right)$ as a triplet $(t)$ with intensity ratio (1:2:1). Besides the methylene group $\left(\mathrm{CH}_{2}\right)$, no other group of protons can have an effect on the active spectrum of the methyl group $\left(\mathrm{CH}_{3}\right)$.

The analysis of methylene group's $\left(\mathrm{CH}_{2}\right){ }^{I} \mathrm{H} N M \mathrm{R}^{\prime}$ 's protons shows the following. The methylene group's protons $\left(\mathrm{CH}_{2}\right)$ are represented as quintet $(q i)$ with the intensity (1:4:6:4:1). This is an abnormity. Protons of methyl $\left(\mathrm{CH}_{3}\right)$ groups must split the signal of methylene group $\left(\mathrm{CH}_{2}\right)$ into four components and form a quartet $(q)$ with an intensity ratio of 1:3:3:1, as based on the spin-spin interaction. In turn, protons of hydroxyl $(O H)$ groups should split each quartet's component of methylene $\left(\mathrm{CH}_{2}\right)$ group into two components to form a double quartet. The signal of methylene $\left(\mathrm{CH}_{2}\right)$ groups should remain as quartet. This happens due to the absence of spin-spin interaction between the hydroxyl $(\mathrm{OH})$ and methylene $\left(\mathrm{CH}_{2}\right)$ groups by the chemical exchange.

The Figures 3-4 shows the proton group's ${ }^{1} H N M R$ spectra's of freshly prepared AAM sample $(0 \mathrm{~h})$ and a sample taken after few days, with an interval of $48 \ldots 72 \mathrm{~h}$ with indication of chemical shift.

Hydroxyl group of protons are represented by two separate peaks (Figure 3) at the time of the initial formation of the AAM and at the time of the functioning of the system $(\tau=0 \mathrm{~h})$. Multiplet component of hydroxyl $(\mathrm{OH})$ proton of ethanol $\left(\mathrm{C}_{2} \mathrm{H}_{5} \mathrm{OH}\right)$ is presented in a form of bulge. The bulge is based in a weak field with a chemical shift of $\delta_{E t O H}=5,32 \mathrm{ppm}$. Signal of water $\left(\mathrm{H}_{2} \mathrm{O}\right)$ protons is presented as an elongated singlet of symmetrical shape with a broad base which is located at $\delta_{H 2 O}=4,71 \mathrm{ppm}$. The difference in chemical shifts of $\mathrm{OH}$ proton $\left(\mathrm{C}_{2} \mathrm{H}_{5} \mathrm{OH}\right)$ and $\mathrm{H}_{2} \mathrm{O}$ proton at this stage $(\tau=0 \mathrm{~h})$ is $\Delta \delta_{l}=0,61 \mathrm{ppm}\left(\Delta f_{l}=244 \mathrm{~Hz}\right)$.

At the initial instant of AAM formation $-\tau=48 \mathrm{~h}$ the presents of two separate signals hydroxyl protons of ethanol and water. Multiplet component of hydroxyl proton of ethanol $(E t O H)$ is presented in a form of bulge with a chemical shift of $\delta_{E t O H}=5,37 \mathrm{ppm}$. Signal of water $\left(\mathrm{H}_{2} \mathrm{O}\right)$ protons is presented as an elongated singlet of symmetrical shape with a broad base which is located at $\delta_{H 2 O}=4,76 \mathrm{ppm}$. The difference in chemical shifts of $\mathrm{OH}$ proton $(E t O H)$ and water $\left(H_{2} O\right)$ proton $-\Delta \delta_{l}=0,61 \mathrm{ppm}\left(\Delta f_{l}=244 \mathrm{~Hz}\right)$.

The spectra from third to sixth $(\tau=120-312 \mathrm{~h})$ is characterized by one summarized peak of hydroxyl protons of ethanol and water. The component of EtOH proton and $\mathrm{H}_{2} \mathrm{O}$ proton is represented by a singlet with the chemical shift of $\delta_{E t O H+H 2 O}=4,74-4,81 \mathrm{ppm}$. The summarized $\mathrm{EtOH}+\mathrm{H}_{2} \mathrm{O}$ protons signal has a nonesymmetric shape with a widened base and a top of regular form.

The analysis of ${ }^{1} H$ NMR spectra of AAM methyl group's protons $\left(\mathrm{CH}_{3}\right)$ states the following $\tau=0$. The protons' methyl group is represented as a quartet $(q)$ with a relative intensity (1:3:3:1) in the initial part of system's operation. This is abnormity as according to Pascal's triangle and on the assumption of protons' methyl group spin-spin interactions, methylene group's $\left(\mathrm{CH}_{3}\right)$ signal has to be split by an adjacent protons' group $\left(\mathrm{CH}_{2}\right)$ as a triplet $(t)$ with intensity ratio $(1: 2: 1)$. Besides the methylene group $\left(\mathrm{CH}_{2}\right)$, no other group of protons can have an effect on the active spectrum of the methyl group $\left(\mathrm{CH}_{3}\right)$. Thus, the methyl group of protons $\left(\mathrm{CH}_{3}\right)$ is located in a strong field with an average value of the chemical shift as $\delta_{\mathrm{CH}_{3}}=1,08 \mathrm{ppm}$. 


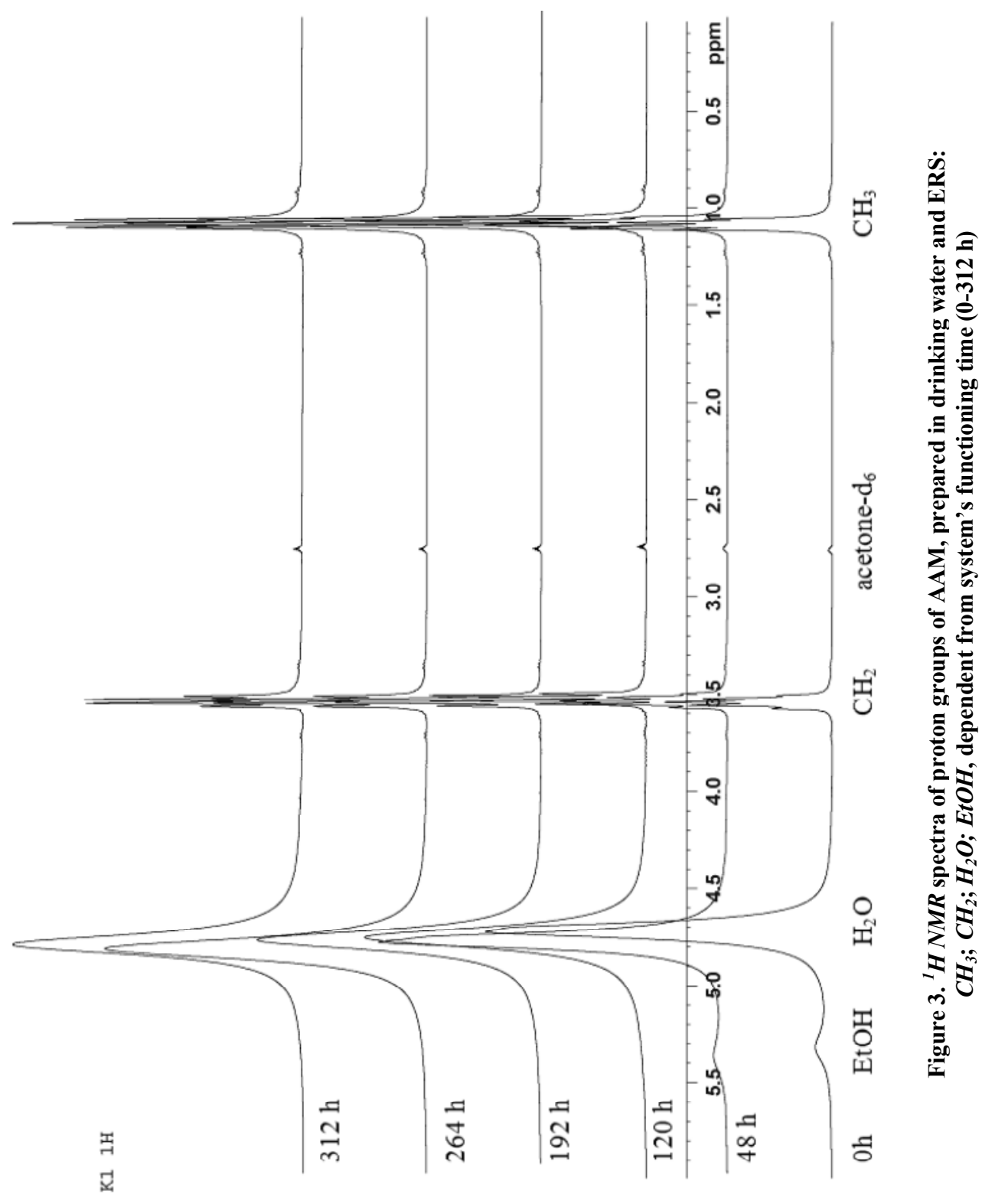

— Ukrainian Food Journal. 2017. Volume 6. Issue 4 —— 693 


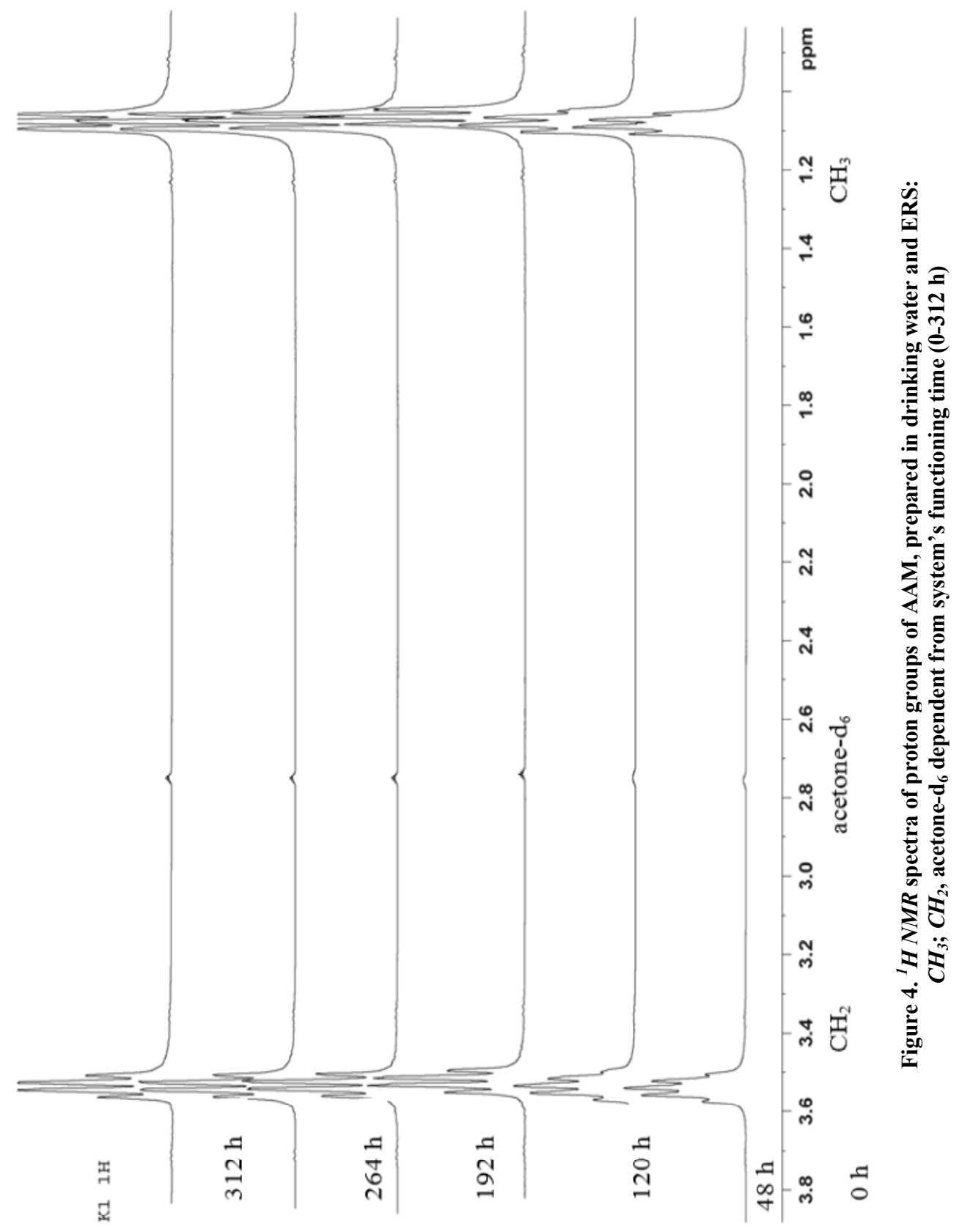


$\tau=48 \mathrm{~h}$. Methyl group of protons $\left(\mathrm{CH}_{3}\right)$ has shifted by a distance of $0,01 \mathrm{ppm}$ towards the strong field from its original position $(\tau=0 \mathrm{~h})$ with an average value of the chemical shift $\delta_{C H 3}=1,07 \mathrm{ppm}$. It has the following individual characteristics of signals' chemical shift's peaks $\delta_{C H 3}=(1,10 ; 1,08 ; 1,06 ; 1,04) \mathrm{ppm}$; distance between the peaks is $8 \mathrm{~Hz}$ relative to each other. Signal's form is quartet $(q)$, which is also an abnormity for the interaction of the above spectrum with methylene group $\left(\mathrm{CH}_{2}\right)$.

$\tau=120 \mathrm{~h}$. Methyl spectrum has shifted by a distance of $0,02 \mathrm{ppm}$ from its original position ( $\tau=0 \mathrm{~h}$ ) with a average value of the chemical shift $\delta_{\mathrm{CH}_{3}}=1,06 \mathrm{ppm}$. Signal's form is triplet $(t)$, which indicates it's stability. This is based on spin-spin interaction with methylene $\left(\mathrm{CH}_{2}\right)$ group's protons. Chemical shift of triplet's individual peaks is $\delta_{\mathrm{CH} 3}=(1,08$; $1,06 ; 1,04) \mathrm{ppm}$.

$\tau=192-312 \mathrm{~h}$. Methyl spectrum has shifted by a distance of $0,01 \mathrm{ppm}$ from its original position ( $\tau=0 \mathrm{~h}$ ) with a average value of the chemical shift $\delta_{C H 3}=1,07 \mathrm{ppm}$. Signal's form is triplet $(t)$, which indicates it's stability. This is based on spin-spin interaction with methylene $\left(\mathrm{CH}_{2}\right)$ group's protons. Chemical shift of triplet's individual peaks is $\delta_{\mathrm{CH} 3}=(1,09$; $1,07 ; 1,05) \mathrm{ppm}$.

The analysis of methylene group's $\left(\mathrm{CH}_{2}\right)^{l} H$ NMR's protons shows the following. The methylene group's protons $\left(\mathrm{CH}_{2}\right)$ are represented as quintet $(q i)$ with the intensity (1:4:6:4:1) at the beginning of AAM's formation process $(\tau=0 \mathrm{~h})$. This is an abnormity. Protons of methyl $\left(\mathrm{CH}_{3}\right)$ groups must split the signal of methylene group $\left(\mathrm{CH}_{2}\right)$ into four components and form a quartet $(q)$ with an intensity ratio of 1:3:3:1, as based on the spinspin interaction. In turn, protons of hydroxyl $(\mathrm{OH})$ groups should split each quartet's component of methylene $\left(\mathrm{CH}_{2}\right)$ group into two components to form a double quartet. The signal of methylene $\left(\mathrm{CH}_{2}\right)$ groups should remain as quartet. This happens due to the absence of spin-spin interaction between the hydroxyl $(\mathrm{OH})$ and methylene $\left(\mathrm{CH}_{2}\right)$ groups by the chemical exchange.

The methylene group of protons $\left(\mathrm{CH}_{2}\right)$ is in a weak field, with the average value of the chemical shift of $\delta_{\mathrm{CH} 2}=3,54 \mathrm{ppm}$, each peak of quartet is located at a distance of $8 \mathrm{~Hz}$ from each other.

The methylene group of protons $\left(\mathrm{CH}_{2}\right)$ has shifted from its original position towards the strong field by $0,01 \mathrm{ppm}$ after the 48 hours. The average value of its chemical shift is $\delta_{\mathrm{CH}_{2}}=3,53 \mathrm{ppm}$. Signal's form is quintet ( $q i$ ). This is an abnormity for the above proton's spectrum.

The first two spectra, in our view, belong to a group with unsteady balance, since the signals' form is abnormal.

Methylene spectrum with an average value of the chemical shift as $\delta_{C H 2}=3,52 \mathrm{ppm}$ has shifted into the strong field with respect to the initial position ( $\tau=0 \mathrm{~h}$ ) by $0,02 \mathrm{ppm}$ after 120 hours expiration. Signal's form is quartet $(q)$, typical for the above proton group, based on the spin-spin interaction with the protons of the methyl $\left(\mathrm{CH}_{3}\right)$ group and chemical exchange between hydroxyl $(\mathrm{OH})$ and methylene $\left(\mathrm{CH}_{2}\right)$ groups. This is the form's stabilization.

Complete structuring of methylene group's $\left(\mathrm{CH}_{2}\right)$ signal takes place approximately after 8 days $(\tau=192 \mathrm{~h})$ : signal's form is quartet $(q)$; location - chemical shift with an average value of $\delta_{\mathrm{CH}_{2}}=3,53 \mathrm{ppm}$, which remains unchanged. The distance between the peaks also remains unchanged $-8 \mathrm{~Hz}$. 


\section{Conclusions}

As a results we have evidence of a complex dynamic of achievement processes of solution equilibrium for AAM prepared in drinking water with $\mathrm{pH}=7,01$ and ERS. At the same time $\mathrm{pH}$ of obtained $\mathrm{AAM}$ is $\mathrm{pH}=8,32$ i.e. alkalescent medium. This value is characterized by a reduced content of free $\mathrm{H}^{+}$ions relative to the $\mathrm{OH}$, i.e. general alkaline reaction of the system. Hydroxyl proton $(\mathrm{OH})$ of ethanol exchange rate is in intermediate area. It happens during the first 48 hours when the concentration of alcohol is constant (strength AAM - 39,94\% vol.) and the system is thermostatic $\left(t=23,5{ }^{\circ} \mathrm{C}\right)$. Signals are separately located. Protons' exchange is accelerated due to the rearrangement of system's structure, during the interval of $\tau=48 \mathrm{~h}$ to $120 \mathrm{~h}$. Since $\tau=120 \mathrm{~h}$, there is only one common signal of mobile protons of asymmetrical shape. The size of chemical shift of the summed signal $\delta_{E t O H+H 2 O}=4,74 \mathrm{ppm}(\tau=120 \mathrm{~h})$ is starting to grow and shifts to the «weak fields» with the value of $\delta_{E t O H+H 2 O}=4,81 \mathrm{ppm}(\tau=312 \mathrm{~h})$.

\section{References}

1. Kawarpal Singh, Bernhard Blümich (2016), NMR spectroscopy with compact instruments, TrAC Trends in Analytical Chemistry, 83, Part A, pp. 12-26.

2. Hore P.J. (2017), NMR Principles, Encyclopedia of Spectroscopy and Spectrometry (Third Edition), pp. 187-194.

3. Latufa Youssouf, Laura Lallemand, Pierre Giraud, Faiza Soulé, Joël Couprie (2017), Ultrasound-assisted extraction and structural characterization by NMR of alginates and carrageenans from seaweeds, Carbohydrate Polymers, 166, pp. 55-63.

4. Gloria del Campo, Juan Zuriarrain, Andoni Zuriarrain, Iñaki Berregi (2016), Quantitative determination of carboxylic acids, amino acids, carbohydrates, ethanol and hydroxymethylfurfural in honey by ${ }^{1} \mathrm{H}$ NMR, Food Chemistry, 196, pp. 1031-1039.

5. Fan Zhu (2017), NMR spectroscopy of starch systems, Food Hydrocolloids, 63, pp. 611624.

6. Yunfei Yuan, Yupeng Zhao, Jiali Yang, Yueming Jiang, Bao Yang (2017), Metabolomic analyses of banana during postharvest senescence by ${ }^{1} \mathrm{H}$-high resolution-NMR, Food Chemistry, 218, pp. 406-412.

7. Cherif Ibrahima Khalil Diop, Hai Long Li, Peng Chen, Bi Jun Xie (2012), Properties of maize starch modified by ball milling in ethanol medium and low field NMR determination of the water molecular mobility in their gels, Journal of Cereal Science, 56 (2), pp. 321331.

8. Ye Tian, Jaana Liimatainen, Aino-Liisa Alanne, Anni Lindstedt, Baoru Yang (2017), Phenolic compounds extracted by acidic aqueous ethanol from berries and leaves of different berry plants, Food Chemistry, 220, pp. 266-281.

9. Sucupira N.R., Alves Filho E.G., Silva L.M.A., E.S. de Brito, Sousa P.H.M. (2017), NMR spectroscopy and chemometrics to evaluate different processing of coconut water, Food Chemistry, 216, pp. 217-224.

10. Bao Qiong Li, Min Li Xu, Xue Wang, Hong Lin Zhai, Jin Jin Liu (2017), An approach to the simultaneous quantitative analysis of metabolites in table wines by ${ }^{1} \mathrm{H}$ NMR selfconstructed three-dimensional spectra, Food Chemistry, 216, pp. 52-59.

11. Elena Shumilina, Rasa Slizyte, Revilija Mozuraityte, Anastasiya Dykyy, Alexander Dikiy (2016), Quality changes of salmon by-products during storage: Assessment and quantification by NMR, Food Chemistry, 211, pp. 803-811. 
12. Alex O. Okaru, Kennedy O. Abuga, Isaac O. Kibwage, Thomas Hausler, Dirk W. Lachenmeier (2017), Aflatoxin contamination in unrecorded beers from Kenya - A health risk beyond ethanol, Food Control, 79, pp. 344-348.

13. Nose A., Hamasaki T., Hojo M. and other (2005), Hydrogen bonding in alcoholic beverages (distilled spirits) and water-ethanol mixture, Journal of agricultural and food chemistry, 53, pp. 7074-7081.

14. Richards S.A., Hollerton J.C. (2011), Essential practical NMR for organic chemistry, Chichester: John Wiley \& Sons, Ltd.

15. Roberts J.D. (2002), Organic chemistry applications, in Grant, D.M., Harris, R.K. (ed.) Encyclopedia of nuclear magnetic resonance, Chichester: John Wiley \& Sons, Ltd, pp. 3386-3400.

16. $\mathrm{Hu}$ N., Wu D., Cross K. and other (2010), Structurability: A collective measure of the structural differences in vodkas, Journal of agricultural and food chemistry, 58, pp. 73947401.

17. Arnold J.T., Dharmatti S.S., Packard M.E. (1951), Chemical effects on nuclear induction signals from organic compounds, Journal of chemical physics, 19, pp. 507.

18. Bloch F, Hansen W.W., Packard M. (1946), The nuclear induction experiment, Physical review, 70, pp. 474-489.

19. Matsugami M., Yamamoto R., Kumai T., Tanaka M., Takamuku T. (2016), Hydrogen bonding in ethanol-water and trifluoroethanol-water mixtures studied by NMR and molecular dynamics simulation, Journal of Molecular Liquids, 217, pp. 3-11.

20. Manazael Z. Jora, Marcus V.C. Cardoso, Edvaldo Sabadini (2017), Correlation between viscosity, diffusion coefficient and spin-spin relaxation rate in ${ }^{1} \mathrm{H}$ NMR of water-alcohols solutions, Journal of Molecular Liquids, 238, pp. 341-346.

21. Kuzmin O., Sujkov S., Topol'nik V. (2013), The change of the hydroxyl protons in aqueous alcoholic mixtures under the process of making vodkas, The advanced science journal, Special issue in China, pp. 15-27.

22. Kuzmin O., Topol'nik V. (2014), Eduction of unsteady equilibrium in vodkas by means of ${ }^{1} \mathrm{H}$ NMR spectroscopy, Advanced science journal, 10, pp. 43-46.

23. Kuzmin O., Suikov S., Niemirich O., Ditrich I., Sylka I. (2017), Effects of the water desalting by reverse osmosis on the process of formation of water-alcohol mixtures. ${ }^{1} \mathrm{H}$ NMR spectroscopy studies, Ukrainian Food Journal, 6(2), pp. 239-257, DOI: 10.24263/2304-974X-2017-6-2-6).

24. Kuzmin O., Suikov S., Koretska I., Matiyashchuk O., Poliovyk V. (2017), Identification of equilibrium state of hydroxyl protons in vodkas by ${ }^{1} \mathrm{H}$ NMR spectroscopy, Ukrainian Food Journal, 6(2), pp. 314-336, DOI: 10.24263/2304-974X-2017-6-1-12). 